\title{
The Fractional Weak Discrepancy of a Partially Ordered Set
}

\author{
Alan Shuchat \\ Department of Mathematics \\ Wellesley College \\ Wellesley, MA 02481 USA \\ Randy Shull \\ Department of Computer Science \\ Wellesley College \\ Wellesley, MA 02481 USA \\ Ann N. Trenk \\ Department of Mathematics \\ Wellesley College \\ Wellesley, MA 02481 USA
}

April 17, 2007

\begin{abstract}
In this paper we introduce the notion of the fractional weak discrepancy of a poset, building on previous work on weak discrepancy in $[?, ?, ?]$. The fractional weak discrepancy $w d_{F}(P)$ of a poset $P=(V, \prec)$ is the minimum nonnegative $k$ for which there exists a function $f: V \rightarrow \mathbf{R}$ satisfying (1) if $a \prec b$ then $f(a)+1 \leq f(b)$ and (2) if $a \| b$ then $|f(a)-f(b)| \leq k$. We formulate the fractional weak discrepancy problem as a linear program and show how its solution can also be used to calculate the (integral) weak discrepancy. We interpret the dual linear program as a circulation problem in a related directed graph and use this to give a structural characterization of the fractional weak discrepancy of a poset. ${ }^{1}$
\end{abstract}

Keywords: weak discrepancy, fractional weak discrepancy

\footnotetext{
${ }^{1}$ The third author's work was supported in part by a Wellesley College Brachman Hoffman Fellowship.
} 


\section{Introduction}

In this paper we consider finite posets $P=(V, \prec)$, where incomparability is denoted by $\|$. We begin with some definitions and background on the (integral) weak discrepancy problem.

Definition 1 The (integral) weak discrepancy of a poset $P=(V, \prec)$ (denoted $w d(P))$ is the least nonnegative integer $k$ for which there exists an integer-valued function $f: V \rightarrow \mathbf{Z}$ satisfying

(1) if $a \prec b$ then $f(a)<f(b)$

("up" constraints)

(2) if $a \| b$ then $|f(a)-f(b)| \leq k$.

Such a labeling is called an optimal weak labeling of $P$ (or of $V$ ).

For example, the poset $R$ in Figure ?? has $w d(R)=2$ with an optimal weak labeling as shown. Definition ?? is motivated by problems like the following. A manager who partially orders her employees by their value to the company needs to assign a salary level to each employee. The "up" constraints ensure that a more valuable employee gets a higher salary than a less valuable one and the "side" constraints are fairness conditions that restrict the salary discrepancies between pairs of incomparable employees. See [?] for additional examples.

If $w d(P) \leq k$, we say that $P$ is $k$-weak. A weak order can be defined as one obtained from a linear order by replacing each element by an antichain. Alternatively, $P=(V, \prec)$ is a weak order if it contains no triple of elements $\{x, y, z\}$ with $x \prec y$ and $z$ incomparable to both $x$ and $y$ [?]. Note that $P$ is 0 -weak if and only if $P$ is a weak order. The weak discrepancy of an order is a measure of how far it is from being a weak order.

The class of $k$-weak orders was introduced in [?]. In that paper a polynomialtime algorithm is presented for recognizing $k$-weak orders, and in the affirmative case, producing an appropriate labeling function. This algorithm can be adapted easily to compute the weak discrepancy in polynomial time, as noted in [?], where weak discrepancy is known as weakness. The main result in [?] is a characterization of weak discrepancy using forcing cycles, which we define later. The related concept of linear discrepancy is studied in [?]. There the function $f$ in Definition ?? is an injection with domain $V$ and can thus be assumed to be a bijection from $V$ to $\{1,2,3, \ldots,|V|\}$.

The definition of fractional weak discrepancy is motivated by further consideration of the salary assignment problem. In the version discussed above, we think of the value $f(a)$ assigned to employee $a$ as a salary level, such as a government salary level. However, for many companies, salaries are not constrained by levels, and can be any dollar amount. It then makes sense to modify (1) to ensure that the salaries assigned to employees $a$ and $b$ are significantly different. This motivates the following definition, in which we can think of $f(a)$ as the salary assigned to employee $a$, and the units of $f(a)$ (e.g., dollars, hundreds of dollars, etc.) should be chosen to make 1 unit a significant salary difference. 

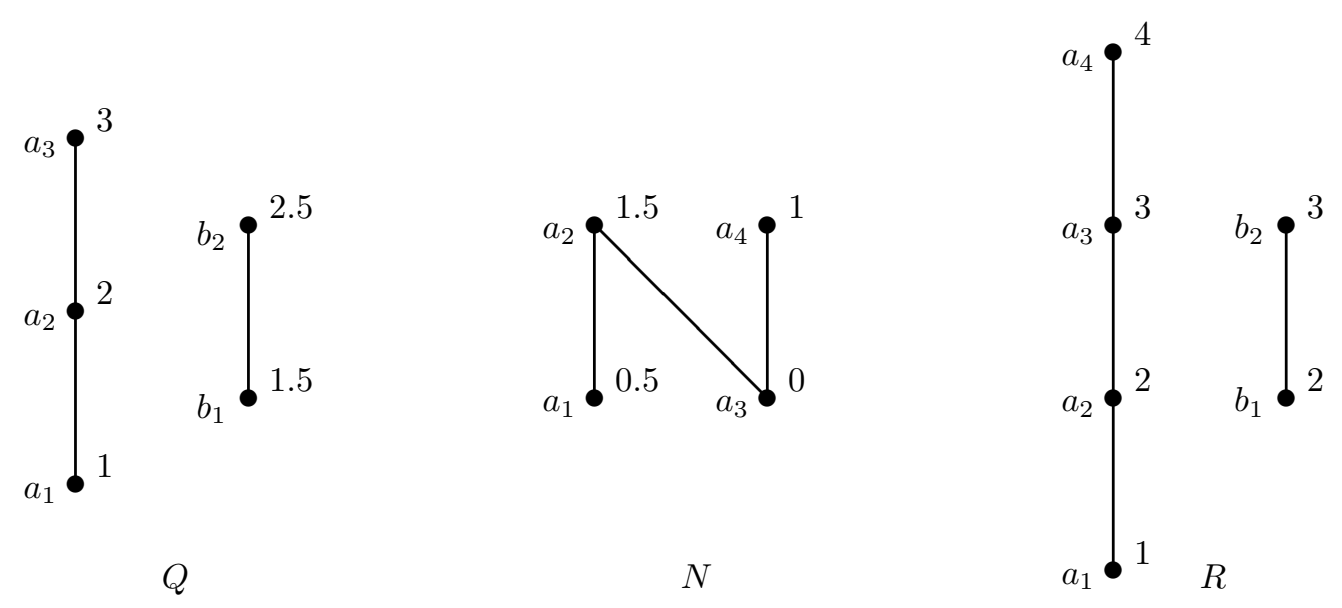

Figure 1: Three posets together with optimal fractional weak labelings.

Definition 2 The fractional weak discrepancy $w_{F}(P)$ of a poset $P=(V, \prec)$ is the minimum nonnegative real number $k$ for which there exists a function $f: V \rightarrow \mathbf{R}$ satisfying

(1) if $a \prec b$ then $f(a)+1 \leq f(b) \quad$ ("up" constraints)

(2) if $a \| b$ then $|f(a)-f(b)| \leq k$. ("side" constraints)

Such a function $f$ is called an optimal fractional weak labeling of $P$ (or of $V)$.

One reason for calling $w d_{F}(P)$ the fractional weak discrepancy is that Remark ?? implies that $w d_{F}(P)$ is rational and that all the values of the labeling function $f$ may be taken to be rational.

Figure ?? illustrates labelings of the posets $Q, N$, and $R$ that show $w d_{F}(Q) \leq$ $1.5, w d_{F}(N) \leq .5$, and $w d_{F}(R) \leq 2$. In Example ?? and Corollary ?? we will prove that in fact these are all equalities. In particular, the poset $R$ is an example for which the weak discrepancy and the fractional weak discrepancy are equal. For posets $Q$ and $N$ we will see that $w d(Q)=2$ and $w d(N)=1$, which are achieved by taking the ceiling of each label given in Figure ??. Equivalently, we could take the floor of each label.

In Proposition ?? we will show that in general by taking the ceiling (or floor) of each label we obtain $w d(P)=\left\lceil w d_{F}(P)\right\rceil$. This establishes a connection between the fractional and (integral) weak discrepancy problems. In the remainder of this paper we will show that both discrepancy problems can be solved using linear programming techniques. Furthermore, we use duality theory to interpret fractional weak discrepancy as a problem about optimal circulations in directed graphs. 


\section{A Linear Programming Approach}

In this section we frame the fractional weak discrepancy problem as a linear program and also study the (integral) weak discrepancy problem in this way.

We will omit the degenerate case of linear orders from further consideration. We now define an LP formulation $P_{F}$ of the fractional weak discrepancy problem in the nondegenerate case.

Definition 3 Given a poset $P=(V, \prec)$ with at least one incomparable pair of elements, let $V=\left\{a_{1}, a_{2}, \ldots, a_{n}\right\}$ and let $P_{F}$ be the following linear program with decision variables $k$ and $x_{1}, x_{2}, \ldots, x_{n}$.

minimize $k$

subject to

$$
\left.\begin{array}{ccc}
x_{i}-x_{j} \leq-1 & \text { for } a_{i} \prec a_{j} & \text { ("up" constraints) } \\
x_{i}-x_{j}-k \leq 0 \\
x_{j}-x_{i}-k \leq 0
\end{array}\right\} \quad \text { for } a_{i} \| a_{j} \quad \text { ("side" constraints) }
$$

Here $x_{i}=f\left(a_{i}\right)$ defines a labeling $f$ of $V$ that satisfies the up and side constraints of Definition ??. Since there is at least one pair of side constraints $x_{i}-x_{j} \leq k, x_{j}-x_{i} \leq k$, or equivalently, $\left|x_{i}-x_{j}\right| \leq k$, each feasible solution has $k \geq 0$ as required in Definition ??.

A feasible solution to $P_{F}$ can be obtained by letting $x_{i}$ be the height of $a_{i}$ in a linear extension of $P$ and letting $k=n-1$, and thus an optimal solution exists to this minimization problem. Since the objective function and the constraints of $P_{F}$ match those of Definition ??, we conclude the following.

Remark 4 In an optimal solution to the linear program $P_{F}, k=w d_{F}(P)$ and the values of $x_{i}$ determine an optimal labeling of $V$.

Given an optimal labeling of $V$, we can add a constant to each $x_{i}$ to produce another optimal labeling in which all labels are nonnegative.

The linear programming formulation allows one to solve fractional weak discrepancy problems using standard implementations of LP algorithms.

Example 5 We formulate the LP for the poset $N$ of Figure ??.

minimize $k$ 
subject to

$$
\begin{aligned}
x_{1}-x_{2} & \leq-1 \\
x_{3}-x_{2} & \leq-1 \\
x_{3}-x_{4} & \leq-1 \\
x_{1}-x_{3}-k & \leq 0 \\
x_{3}-x_{1}-k & \leq 0 \\
x_{1}-x_{4}-k & \leq 0 \\
x_{4}-x_{1}-k & \leq 0 \\
x_{2}-x_{4}-k & \leq 0 \\
x_{4}-x_{2}-k & \leq 0 \\
x_{1}, \ldots, x_{4}, k & \text { unrestricted }
\end{aligned}
$$

The LP package LINGO [?] produces $x_{1}=.5, x_{2}=1.5, x_{3}=0, x_{4}=1$, $k=.5$ as an optimal solution to this problem. It is easy to verify that this labeling, which was given in Figure ??, is a feasible solution to the LP. We will prove it is optimal in Example ??. As we can see from Example ??, even a small poset can yield a large number of constraints. One can check that in posets where there are chains of three or more elements, we do not need to include inequalities for comparabilities that are implied by transitivity, such as $a_{1} \prec a_{3}$ in poset $Q$.

The following remark follows directly from Remark ?? and Definitions ?? and ??.

Remark 6 The (integral) weak discrepancy problem of Definition ?? can be formulated as an integer program $P_{I}$ by restricting all the variables in $P_{F}$ to be integers.

We end this section with a proposition that shows how a solution to the linear program $P_{F}$ yields a solution to the integer program $P_{I}$. This means that the weak discrepancy of a poset can be found using standard implementations of LP algorithms.

Proposition 7 Let $P=(V, \prec)$ be a poset with $V=\left\{a_{1}, a_{2}, \ldots, a_{n}\right\}$ and let $x_{1}, x_{2}, \ldots, x_{n}, k$ be an optimal solution to the fractional weak discrepancy problem $P_{F}$. Then $\left\lceil x_{1}\right\rceil,\left\lceil x_{2}\right\rceil, \ldots,\left\lceil x_{n}\right\rceil,\lceil k\rceil$ is an optimal solution to the weak discrepancy problem $P_{I}$.

Proof. First we show that $\left\lceil x_{1}\right\rceil,\left\lceil x_{2}\right\rceil, \ldots,\left\lceil x_{n}\right\rceil,\lceil k\rceil$ defines a feasible solution to $P_{I}$. For each up constraint, $x_{i} \leq x_{j}-1 \leq\left\lceil x_{j}\right\rceil-1$, thus $\left\lceil x_{i}\right\rceil \leq\left\lceil x_{j}\right\rceil-1$. For each side constraint, $x_{i} \leq x_{j}+k \leq\left\lceil x_{j}\right\rceil+\lceil k\rceil$, thus $\left\lceil x_{i}\right\rceil \leq\left\lceil x_{j}\right\rceil+\lceil k\rceil$. In fact, this is an optimal solution to $P_{I}$, since otherwise there would be a feasible solution to $P_{I}$, and hence to $P_{F}$, with objective function value $k^{\prime} \leq\lceil k\rceil-1<k$. 


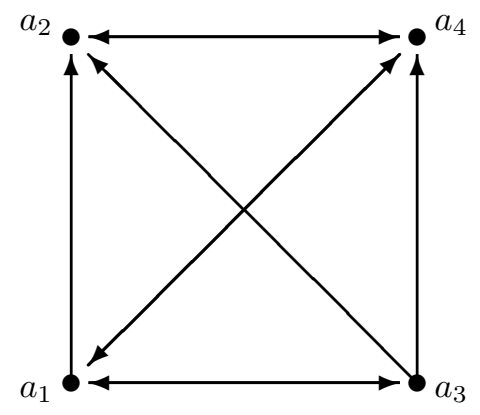

Figure 2: The preference-indifference digraph $\vec{G}_{N}$.

\section{Forcing Cycles and the Dual Program}

\subsection{Forcing cycles and the weak discrepancy problem}

In [?], the weak discrepancy is characterized using forcing cycles. A forcing cycle $C$ of poset $P=(V, \prec)$ is a sequence $C: a_{0}, a_{1}, \ldots, a_{m}=a_{0}$ of $m \geq 2$ elements of $V$ for which $a_{i} \prec a_{i+1}$ or $a_{i} \| a_{i+1}$ for each $i: 0 \leq i<m$. In [?], these are called picycles (preference-indifference cycles).

If $C$ is a forcing cycle, we write $u p(C)=\left|\left\{i: a_{i} \prec a_{i+1}\right\}\right|$ and $\operatorname{side}(C)=\mid\{i$ : $\left.a_{i} \| a_{i+1}\right\} \mid$. For example, the poset $Q$ of Figure ?? has forcing cycle $C: a_{1} \prec$ $a_{2} \prec a_{3}\left\|b_{1} \prec b_{2}\right\| a_{1}$ with $u p(C)=3$, side $(C)=2$ and $w d_{F}(Q)=3 / 2$. (As noted in Section ?? we will prove later that the values we claim for $w d_{F}$ of $Q, N$, and $R$ are correct.) Similarly, the poset $N$ has forcing cycle $C: a_{1} \prec a_{2}\left\|a_{4}\right\| a_{1}$ with $u p(C)=1$, side $(C)=2$ and $w d_{F}(N)=1 / 2$. Also, the poset $R$ has forcing cycle $C: a_{1} \prec a_{2} \prec a_{3} \prec a_{4}\left\|b_{1} \prec b_{2}\right\| a_{1}$ with $u p(C)=4$, side $(C)=2$ and $w d_{F}(R)=4 / 2=2$.

In [?], Gimbel and Trenk prove that for all posets $P, w d(P)=\max _{C}\left\lceil\frac{u p(C)}{\operatorname{side}(C)}\right\rceil$ where the maximum is taken over all forcing cycles $C$ in $P$. The analogous result for fractional weak discrepancy appears in Theorem ?? and involves a directed graph $\vec{G}_{P}$ associated with poset $P$.

Given a poset $P=(V, \prec)$ with at least one incomparable pair, we define the preference-indifference digraph $\vec{G}_{P}=(V, E)$ with arc set $E=U \cup S$, where $U=\{(a, b): a \prec b\}$ and $S=\{(a, b): a \| b\}$. We call $U$ the set of "up" $\operatorname{arcs}$ and $S$ the set of "side" $\operatorname{arcs}$ in $\vec{G}_{P}$. Figure ?? illustrates $\vec{G}_{N}$ for the poset $N$ of Figure ??.

Notice that the forcing cycles in a poset $P$ correspond precisely to the directed cycles in the digraph $\vec{G}_{P}$. Furthermore, we make the following observation about directed cycles in $\vec{G}_{P}$.

Remark 8 Since each poset $P$ that we consider has at least one incomparable pair, $\vec{G}_{P}$ has at least one directed cycle, and since precedence $(\prec)$ is transitive, every directed cycle of $\vec{G}_{P}$ must contain at least two side arcs. 


\subsection{Duality}

In this section, we interpret the dual $D_{F}$ of the linear program $P_{F}$ as a circulation problem on the directed graph $\vec{G}_{P}$. We also establish a connection between circulation flow and forcing cycles and use linear programming duality to solve the fractional weak discrepancy problem.

A circulation is a flow in a directed graph where flow is conserved at each vertex. More formally, a circulation on a digraph $\vec{G}=(V, E)$ is a function $\Phi: E \rightarrow \mathbf{R}$ satisfying the equation

$$
\sum_{\{b:(a, b) \in E\}} \Phi(a, b)-\sum_{\{b:(b, a) \in E\}} \Phi(b, a)=0 \quad \text { for each } a \in V .
$$

For example, the circulation equations for the digraph $\vec{G}_{N}$ shown in Figure ?? take the following form. Here we use the vertex-arc incidence matrix representation of $\vec{G}_{N}$, each $u_{i j}$ is the flow $\Phi(i, j)$ along the up arc $(i, j) \in U$ and, similarly, each $s_{i j}$ is the flow along the side $\operatorname{arc}(i, j) \in S$.

$$
\left(\begin{array}{ccc|cccccc}
1 & 0 & 0 & 1 & -1 & 1 & -1 & 0 & 0 \\
-1 & -1 & 0 & 0 & 0 & 0 & 0 & 1 & -1 \\
0 & 1 & 1 & -1 & 1 & 0 & 0 & 0 & 0 \\
0 & 0 & -1 & 0 & 0 & -1 & 1 & -1 & 1
\end{array}\right)\left(\begin{array}{l}
u_{12} \\
u_{32} \\
u_{34} \\
s_{13} \\
s_{31} \\
s_{14} \\
s_{41} \\
s_{24} \\
s_{42}
\end{array}\right)=\left(\begin{array}{c}
0 \\
0 \\
0 \\
0
\end{array}\right)
$$

In the proof of Theorem ?? we will need to apply the following well-known result about circulations, which appears in [?].

Theorem 9 (Flow Decomposition Theorem) Every circulation on a digraph $\vec{G}=(V, E)$ can be decomposed into nonnegative flows along at most $|E|$ directed cycles.

Proposition 10 Suppose $P$ is a poset with at least one incomparable pair. The dual $D_{F}$ of the linear program $P_{F}$ can be interpreted as an optimization problem on the digraph $\vec{G}_{P}$ in which we wish to find a circulation that maximizes the sum of the flows along the up arcs while constraining the sum of the flows along the side arcs to equal one.

Proof. Given poset $P=(V, \prec)$ with at least one incomparable pair, let $P_{F}$ be the LP in Definition ??. Let $n=|V|, m=|U|$, and $2 p=|S|$, where $U$ (resp. $S$ ) is the set of up arcs (resp. side arcs) in $\vec{G}_{P}$, and let $\mathbf{e}_{m}=(1, \ldots, 1)^{T} \in \mathbf{R}^{m}$. We denote the vector of primal variables by $\mathbf{x}=\left(x_{1}, \ldots, x_{n}\right)^{T} \in \mathbf{R}^{n}$ and let $\mathbf{u}^{\prime} \in \mathbf{R}^{m}$ and $\mathbf{s}^{\prime} \in \mathbf{R}^{2 p}$ be the (column) vectors of dual variables corresponding to 
the up and side constraints of $P_{F}$, respectively. Finally, let matrix $A$ be the $m \times n$ matrix of coefficients of the variables $x_{i}$ in the up constraints of Definition ??, and let $B$ be the corresponding $2 p \times n$ matrix for the side constraints. Then we can express $P_{F}$ and its dual program $D_{F}$ in the following form.

$$
\begin{array}{cr}
P_{F}: \min k & D_{F}: \max -\mathbf{e}_{m} \cdot \mathbf{u}^{\prime} \\
\text { subject to } & \text { subject to } \\
A \mathbf{x} \leq-\mathbf{e}_{m} & A^{T} \mathbf{u}^{\prime}+B^{T} \mathbf{s}^{\prime}=\mathbf{0}_{n} \\
B \mathbf{x}-k \mathbf{e}_{2 p} \leq \mathbf{0}_{2 p} & -\mathbf{e}_{2 p} \cdot \mathbf{s}^{\prime}=1 \\
\mathbf{x}, k \text { unrestricted } & \mathbf{u}^{\prime} \leq \mathbf{0}_{m}, \mathbf{s}^{\prime} \leq \mathbf{0}_{2 p}
\end{array}
$$

This is illustrated in Example ?? below. In matrix form, the constraints in $P_{F}$ and $D_{F}$ are the following.

$$
\left(\begin{array}{cc}
A & \mathbf{0}_{m} \\
B & -\mathbf{e}_{2 p}
\end{array}\right)\left(\begin{array}{l}
\mathbf{x} \\
k
\end{array}\right) \leq\left(\begin{array}{c}
-\mathbf{e}_{m} \\
\mathbf{0}_{2 p}
\end{array}\right) \quad\left(\begin{array}{cc}
A^{T} & B^{T} \\
\mathbf{0}_{m}^{T} & -\mathbf{e}_{2 p}^{T}
\end{array}\right)\left(\begin{array}{c}
\mathbf{u}^{\prime} \\
\mathbf{s}^{\prime}
\end{array}\right)=\left(\begin{array}{c}
\mathbf{0}_{n} \\
1
\end{array}\right)
$$

Now we let $\mathbf{u}=-\mathbf{u}^{\prime}$ and $\mathbf{s}=-\mathbf{s}^{\prime}$, so that the dual is as follows.

$$
\begin{gathered}
D_{F}: \max \mathbf{e}_{m} \cdot \mathbf{u} \\
A^{T} \mathbf{u}+B^{T} \mathbf{s}=\mathbf{0}_{n} \\
\mathbf{e}_{2 p} \cdot \mathbf{s}=1 \\
\mathbf{u} \geq \mathbf{0}_{m}, \quad \mathbf{s} \geq \mathbf{0}_{2 p}
\end{gathered}
$$

In matrix form, the dual constraints after the sign change are given below.

$$
\left(\begin{array}{ll}
A^{T} & B^{T} \\
\mathbf{0}_{m}^{T} & \mathbf{e}_{2 p}^{T}
\end{array}\right)\left(\begin{array}{l}
\mathbf{u} \\
\mathbf{s}
\end{array}\right)=\left(\begin{array}{c}
\mathbf{0}_{n} \\
1
\end{array}\right)
$$

We can interpret the coordinates of $\mathbf{u}$ and $\mathbf{s}$ as nonnegative flows along the arcs of $\vec{G}_{P}$. Each of the $n$ rows of $A^{T}, B^{T}$ corresponds to a vertex of $\vec{G}_{P}$, with a 1 for every arc leaving the vertex and a -1 for every arc entering it as illustrated in Equation (??) above. So $A^{T} \mathbf{u}+B^{T} \mathbf{s}=\mathbf{0}_{n}$ states that flow is preserved at each vertex of $\vec{G}_{P}$, and a feasible solution is a circulation on $\vec{G}_{P}$ subject to the additional constraint that the sum of the flows on the side $\operatorname{arcs}, \mathbf{e}_{2 p} \cdot \mathbf{s}$, equals 1. The objective is to maximize $\mathbf{e}_{m} \cdot \mathbf{u}$, which is just the sum of the flows on the up arcs.

Recall that the optimal value for the primal problem $P_{F}$ is $w d_{F}(P)$, by Remark ??. Since at optimality the primal and dual problems have the same objective function value, the maximum value of $\mathbf{e}_{m} \cdot \mathbf{u}$ equals $w d_{F}(P)$. This proves the following corollary. 
Corollary 11 Suppose $P$ is a poset with at least one incomparable pair. Then $w d_{F}(P)$ is the maximum sum of the flows along the up arcs taken over all circulations in $\vec{G}_{P}$ for which the sum of the flows along the side arcs equals one.

Example 12 In Example ??, we show $P_{F}$ for the poset $N$. Here we express $P_{F}$ in matrix form. Each row corresponds to an arc of $\vec{G}_{N}$ and each column (except for the last one) corresponds to a vertex.

$$
P_{F}: \min k
$$

subject to

$$
\left.\begin{array}{cccc|c}
1 & -1 & 0 & 0 & 0 \\
0 & -1 & 1 & 0 & 0 \\
0 & 0 & 1 & -1 & 0 \\
\hline 1 & 0 & -1 & 0 & -1 \\
-1 & 0 & 1 & 0 & -1 \\
1 & 0 & 0 & -1 & -1 \\
-1 & 0 & 0 & 1 & -1 \\
0 & 1 & 0 & -1 & -1 \\
0 & -1 & 0 & 1 & -1
\end{array}\right)\left(\begin{array}{c}
x_{1} \\
x_{2} \\
x_{3} \\
x_{4} \\
k
\end{array}\right) \leq\left(\begin{array}{c}
-1 \\
-1 \\
-1 \\
0 \\
0 \\
0 \\
0 \\
0 \\
0
\end{array}\right)
$$

Let the dual variables (after the sign change) be $u_{i j}$ for the $m=3$ up constraints $x_{i}-x_{j} \leq-1$ and $s_{i j}$ for the $2 p=6$ side constraints $x_{i}-x_{j}-k \leq 0$. We express $D_{F}$ in matrix form below. Now each row of the dual matrix (except the last) corresponds to a vertex of $\vec{G}_{N}$ and each column corresponds to an arc. This part of the matrix is identical to the coefficient matrix in Equation (??) and corresponds to the partitioned matrix $\left(A^{T} B^{T}\right)$ of Equation (??).

$$
\begin{aligned}
& D_{F}: \max u_{12}+u_{32}+u_{34} \\
& \text { subject to }
\end{aligned}
$$

$$
\begin{gathered}
\left(\begin{array}{ccc|cccccc}
1 & 0 & 0 & 1 & -1 & 1 & -1 & 0 & 0 \\
-1 & -1 & 0 & 0 & 0 & 0 & 0 & 1 & -1 \\
0 & 1 & 1 & -1 & 1 & 0 & 0 & 0 & 0 \\
0 & 0 & -1 & 0 & 0 & -1 & 1 & -1 & 1 \\
\hline 0 & 0 & 0 & 1 & 1 & 1 & 1 & 1 & 1
\end{array}\right)\left(\begin{array}{l}
u_{12} \\
u_{32} \\
u_{34} \\
s_{13} \\
s_{31} \\
s_{14} \\
s_{41} \\
s_{24} \\
s_{42}
\end{array}\right)=\left(\begin{array}{l}
0 \\
0 \\
0 \\
0 \\
1
\end{array}\right) \\
\mathbf{u} \geq \mathbf{0}_{3}, \mathbf{s} \geq \mathbf{0}_{6}
\end{gathered}
$$

One feasible solution is $u_{12}=s_{24}=s_{41}=0.5$, with all other dual variables equal to 0 , i.e., flow is preserved at each of the $n=4$ vertices and the flows along the side arcs sum to 1 . The sum of the flows along the up arcs is 0.5 , which is the value of $k$ found for the solution to the primal problem in Example ??. Since the primal and dual objective values are the same, these must be the optimal solutions to the two problems and so $w d_{F}(N)=0.5$. 


\subsection{Forcing cycles and the fractional weak discrepancy problem}

We are now ready to prove an analogue to Gimbel and Trenk's theorem in [?] that characterizes weak discrepancy using forcing cycles. Our approach uses duality theory and circulations. An alternative approach can be based on a result of Bellman (see [?]) about shortest paths and constraint digraphs.

Theorem 13 Let $P=(V, \prec)$ be a poset with at least one incomparable pair. Then $w d_{F}(P)=\max _{C} \frac{u p(C)}{\text { side }(C)}$, taken over all forcing cycles $C$ in $P$. Any forcing cycle $C_{0}$ that achieves the maximum determines an optimal solution to $D_{F}$ in which the nonzero variables are all equal to $\frac{1}{\operatorname{side}\left(C_{0}\right)}$ and are the flows along the arcs of the corresponding directed cycle in $\vec{G}_{P}$.

Proof. Consider an optimal solution to $D_{F}$, with dual variables $u_{i j}, s_{i j}$ corresponding to flows along the up $\operatorname{arcs}(i, j) \in U$ and the side $\operatorname{arcs}(i, j) \in S$. By Proposition ??, this solution is a circulation that maximizes $\sum u_{i j}$ subject to the restriction $\sum s_{i j}=1$. This circulation can be represented as cycle flow along at most $\left|E\left(\vec{G}_{P}\right)\right|$ directed cycles $C_{1}, C_{2}, \ldots, C_{r}$, using Theorem ??. By Remark ?? forcing cycles exist in $P$, and since $P$ is finite some cycle $C_{0}$ achieves the maximum $\max _{C} \frac{u p(C)}{\operatorname{side}(C)}$ among all directed cycles $C$ of $\vec{G}_{P}$. We show an optimal solution can be achieved by restricting all (nonzero) flow to $C_{0}$. We accomplish this by iteratively moving the flow from $C_{j}$ to $C_{0}$ for $j=1$ to $r$.

Repeat the following argument for $j=1$ to $r$. Let

$$
\varepsilon_{j}=\frac{\operatorname{side}\left(C_{j}\right)}{u p\left(C_{j}\right)}-\frac{\operatorname{side}\left(C_{0}\right)}{u p\left(C_{0}\right)} .
$$

Note that $\varepsilon_{j} \geq 0$ since $\frac{u p\left(C_{j}\right)}{\operatorname{side}\left(C_{j}\right)} \leq \frac{u p\left(C_{0}\right)}{\operatorname{side}\left(C_{0}\right)}$. Solving for $\operatorname{side}\left(C_{j}\right)$, we obtain

$$
\operatorname{side}\left(C_{j}\right)=u p\left(C_{j}\right) \cdot\left[\frac{\operatorname{side}\left(C_{0}\right)}{u p\left(C_{0}\right)}+\varepsilon_{j}\right] .
$$

Let $f_{j}$ be the flow around $C_{j}$. Define a new flow by removing $f_{j}$ from each arc of the directed cycle $C_{j}$ and adding

$$
f_{j} \cdot\left[\frac{u p\left(C_{j}\right)}{u p\left(C_{0}\right)}+\frac{\varepsilon_{j} \cdot u p\left(C_{j}\right)}{\operatorname{side}\left(C_{0}\right)}\right] \geq 0
$$

to each arc of $C_{0}$.

We show that the resulting change in the dual variables also yields an optimal solution to $D_{F}$. First we establish feasibility. The conservation constraints are maintained when we remove the same value from the flow along each arc of the directed cycle $C_{j}$, and similarly when we add the same value to the flow along each $\operatorname{arc}$ of $C_{0}$. The sum of the flows along the side $\operatorname{arcs}$ of $\vec{G}_{P}$ has not changed 
since we have removed a total of $f_{j} \cdot \operatorname{side}\left(C_{j}\right)$ from the side $\operatorname{arcs}$ of $C_{j}$, but by Equation (??) have added a total of

$$
\begin{aligned}
f_{j} \cdot\left[\frac{u p\left(C_{j}\right)}{u p\left(C_{0}\right)}+\frac{\varepsilon_{j} \cdot u p\left(C_{j}\right)}{\operatorname{side}\left(C_{0}\right)}\right] \cdot \operatorname{side}\left(C_{0}\right) & =f_{j} \cdot u p\left(C_{j}\right) \cdot\left[\frac{\operatorname{side}\left(C_{0}\right)}{u p\left(C_{0}\right)}+\varepsilon_{j}\right] \\
& =f_{j} \cdot \operatorname{side}\left(C_{j}\right)
\end{aligned}
$$

to the side arcs of $C_{0}$. Thus, the new flow values yield a feasible solution to $D_{F}$.

The value of the objective function, $\sum u_{i j}$, has not decreased since we removed a total of $f_{j} \cdot u p\left(C_{j}\right)$ from the up arcs of $C_{j}$ and added a total of

$$
\begin{aligned}
f_{j} \cdot\left[\frac{u p\left(C_{j}\right)}{u p\left(C_{0}\right)}+\frac{\varepsilon_{j} \cdot u p\left(C_{j}\right)}{\operatorname{side}\left(C_{0}\right)}\right] \cdot u p\left(C_{0}\right) & =f_{j} \cdot u p\left(C_{j}\right)\left[1+\frac{\varepsilon_{j} \cdot u p\left(C_{0}\right)}{\operatorname{side}\left(C_{0}\right)}\right] \\
& \geq f_{j} \cdot u p\left(C_{j}\right)
\end{aligned}
$$

to the up arcs of $C_{0}$. Thus the new flow assignment yields an optimal solution to $D_{F}$. Once we complete this process for $j=r$, we have an optimal solution to the circulation problem $D_{F}$ in which all nonzero flow is along arcs in a single directed cycle $C_{0}$ that achieves $\max _{C} \frac{u p(C)}{s i d e(C)}$ among all directed cycles $C$ of $\vec{G}_{P}$. The conservation constraints imply that the flows along each arc of $C_{0}$ are equal, i.e., there exists some $v \geq 0$ such that

$$
u_{i j}\left(\text { resp., } s_{i j}\right)= \begin{cases}v & \text { if }(i, j) \in U(\text { resp. }, S) \text { is an arc of } C_{0} \\ 0 & \text { otherwise }\end{cases}
$$

This equation restricted to side $\operatorname{arcs}$ implies $v \cdot \operatorname{side}\left(C_{0}\right)=\sum s_{i j}=1$, so that $v=\frac{1}{\operatorname{side}\left(C_{0}\right)}$. At optimality the values of the objective functions of $P_{F}$ and its dual $D_{F}$ are equal. The value of the former equals $w d_{F}(P)$. Thus,

$$
\begin{aligned}
w d_{F}(P) & =\sum u_{i j} \\
& =v \cdot u p\left(C_{0}\right) \\
& =\frac{u p\left(C_{0}\right)}{\operatorname{side}\left(C_{0}\right)} \\
& =\max _{C} \frac{u p(C)}{\operatorname{side}(C)}
\end{aligned}
$$

as required.

Example 14 We continue analyzing the primal and dual problems for the poset $N$ shown in Figure ??. We apply Theorem ?? to the poset $N$, continuing our results from Examples ?? and ??. One can check that the forcing cycle $C$ : $a_{1} \prec a_{2}\left\|a_{4}\right\| a_{1}$ with $u p(C)=1$ and $\operatorname{side}(C)=2$ achieves $\max _{C} \frac{u p(C)}{\operatorname{side}(C)}=\frac{1}{2}$. As stated in Theorem ??, the forcing cycle $C$ determines an optimal solution to $D_{F}$, namely $u_{12}=s_{24}=s_{41}=0.5$ with all other dual variables equaling 0 . This is precisely the optimal solution found in Example ??. 
For some posets such as $Q$ and $R$ of Figure ??, it is easy to identify the forcing cycles $C$ that achieve the maximum ratio of up $(C)$ to side $(C)$. We denote the poset $Q$ of Figure ?? by $\mathbf{3}+\mathbf{2}$ and $R$ by $\mathbf{4 + 2}$, and more generally write $\mathbf{r}_{\mathbf{1}}+\mathbf{r}_{\mathbf{2}}+\cdots+\mathbf{r}_{\mathbf{t}}$ with $r_{1} \geq r_{2} \geq \cdots \geq r_{t}$ for the poset consisting of the disjoint union of $t$ chains with $r_{i}$ elements in the $i$ th chain. As observed in [?], a forcing cycle $C$ in this poset with largest ratio of $u p(C)$ to side $(C)$ is obtained by cycling through the subposet $\mathbf{r}_{1}+\mathbf{r}_{\mathbf{2}}$, thus proving the following corollary to Theorem ??. In particular, this establishes $w d_{F}(Q)=1.5$ and $w d_{F}(R)=2$, as claimed in Section ??.

Corollary 15 If $P$ is the disjoint union $\mathbf{r}_{\mathbf{1}}+\mathbf{r}_{\mathbf{2}}+\cdots+\mathbf{r}_{\mathbf{t}}$ of $t \geq 2$ chains with $r_{1} \geq r_{2} \geq \cdots \geq r_{t}$, then $w d_{F}(P)=\left(\frac{r_{1}+r_{2}}{2}\right)-1$.

\section{Conclusion}

In this paper we have explored some of the connections between weak discrepancy and fractional weak discrepancy. Proposition ?? gives a way to solve the weak discrepancy problem by rounding up an optimal solution to the corresponding fractional weak discrepancy problem. Theorem ??, characterizing fractional weak discrepancy using forcing cycles, is an analogue of Theorem 2.1 of [?], and Corollary ?? is an analogue of Theorem 21 in [?].

Several additional results in [?] and [?] about weak discrepancy have fractional weak discrepancy counterparts. We mention two such results below, one giving an upper bound on fractional weak discrepancy and the other calculating the fractional weak discrepancy for the standard example posets $S_{n}$. Theorem ?? is an analogue of Theorem 12 in [?], whose proof can be adapted easily to the fractional case.

Theorem 16 If $P$ is an n-element poset, then $0 \leq w d_{F}(P) \leq \frac{n-2}{2}$. Moreover, the lower bound is achieved precisely when $P$ is a weak order and the upper bound is achieved precisely when $P$ is the disjoint union of two chains.

The standard example $S_{n}=(V, \prec)$ for $n \geq 2$ is the height two poset with $V=\left\{a_{1}, a_{2}, \ldots, a_{n}\right\} \cup\left\{b_{1}, b_{2}, \ldots, b_{n}\right\}$ whose only comparabilities are $a_{i} \prec b_{j}$ for $i \neq j$. This poset has the forcing cycle $C: a_{1} \prec b_{2}\left\|a_{2} \prec b_{1}\right\| a_{1}$ with $\frac{u p(C)}{\operatorname{side}(C)}=1$, thus $w d_{F}\left(S_{n}\right) \geq 1$ by Theorem ??. The labeling $f\left(a_{i}\right)=1$, $f\left(b_{i}\right)=2$ for $i: 1 \leq i \leq n$, shows $w d_{F}\left(S_{n}\right) \leq 1$. This proves the following analogue of Theorem 19 in [?].

Theorem 17 For all $n \geq 2, w d_{F}\left(S_{n}\right)=w d\left(S_{n}\right)=1$.

Results like Theorems ?? and ?? are analogues of theorems already known for weak discrepancy. In future work [?] and [?] we use the more refined measure $w d_{F}(P)$ to distinguish among classes of posets having the same $w d(P)$. Note that the current paper predates [?] and [?]. 


\section{References}

[1] R. Ahuja, T. Magnanti, and J. Orlin. Network flows. Prentice Hall, Englewood Cliffs, NJ, 1993.

[2] K.P. Bogart. Introductory Combinatorics. Harcourt Brace Jovanovich, New York, 1990.

[3] T. Cormen, C. Leiserson, R. Rivest, and C. Stein. Introduction to Algorithms. The MIT Press, Cambridge, Massachusetts, 2001.

[4] P.C. Fishburn. Interval orders and interval graphs: A study of partially ordered sets. John Wiley \& Sons, New York, 1985.

[5] J.G. Gimbel and A.N. Trenk. On the weakness of an ordered set. SIAM J. Discrete Math., 11:655-663, 1998.

[6] Lindo Systems, Chicago. LINGO 7.0, 2002.

[7] A. Shuchat, R. Shull, and A. Trenk. Range of the fractional weak discrepancy function. ORDER, 23:51-63, 2006.

[8] A. Shuchat, R. Shull, and A. Trenk. Fractional weak discrepancy of posets and certain forbidden configurations. Submitted.

[9] P.J. Tanenbaum, A.N. Trenk, and P.C. Fishburn. Linear discrepancy and weak discrepancy of partially ordered sets. ORDER, 18:201-225, 2001.

[10] A.N. Trenk. On $k$-weak orders: Recognition and a tolerance result. Discrete Math., 181:223-237, 1998. 